\title{
Managing maturity states in a collaborative platform for the iDMU of aeronautical assembly lines
}

\author{
D. Morales-Palma ${ }^{1 *}$, I. Eguía ${ }^{2}$, M. Oliva ${ }^{3}$, F. Mas $^{3}$, C. Vallellano ${ }^{1}$ \\ ${ }^{1}$ Dpt. Mechanical Engineering and Manufacturing, University of Seville, Seville, Spain \\ ${ }^{2}$ Dpt. Industrial Management, University of Seville, Seville, Spain \\ ${ }^{3}$ Airbus Group, Seville, Spain \\ dmpalma@us.es, ies@us.es, manuel.oliva@airbus.com, \\ fernando.maseairbus.com, carpofor@us.es
}

\begin{abstract}
Collaborative Engineering aims to integrate both functional and industrial design. This goal requires integrating the design processes, the design teams and using a single common software platform to hold all the stakeholders contributions. Airbus company coined the concept of the industrial Digital Mock Up (iDMU) as the necessary unique deliverable to perform the design process with a unique team. Previous virtual manufacturing projects confirmed the potential of the iDMU to improve the industrial design process in a collaborative engineering environment. This paper presents the methodology and preliminary results for the management of the maturity states of the iDMU with all product, process and resource information associated with the assembly of an aeronautical component. The methodology aims to evaluate the suitability of a PLM platform to implement the iDMU in the creation of a control mechanism that allows a collaborative work.
\end{abstract}

Keywords: Product and process maturity · Industrial Digital Mock-Up (iDMU)

- Digital manufacturing · Digital factory $\cdot$ PLM

\section{Introduction}

Reducing product development time, costs and quality problems can be achieve through effective collaboration across distributed and multidisciplinary design teams. This collaboration requires a computational framework which effectively enables capture, representation, retrieval and reuse of product knowledge. Product Lifecycle Management (PLM) refers to this enabling framework to help connect, organize, control, manage, track, consolidate and centralize all the mission-critical information that affects a product and the associate processes and resources. PLM offers a process to streamline collaboration and communication between product stakeholders, engineering, design, manufacturing, quality and other key disciplines.

Collaboration between product and process design teams has the following advantages for the company: reduction of time required to perform tasks; improvement of the ability to solve complex problems; increase of the ability to generate creative alternatives; discussion of each alternative to select as viable and to make decisions;

adfa, p. 1, 2011.

(C) Springer-Verlag Berlin Heidelberg 2011 
communication improvement; learning; personal satisfaction; and encouraging innovation [1]. However, collaboration processes need to be explicitly designed and managed to maximize the positive results of such an effort.

Group interaction and cooperation requires four aspects to be considered: people have to exchange information (communication), organize the work (coordination), operate together in a collective workspace (group memory) and be informed about what is happening and get the necessary information (awareness).

Maturity models have been designed to assess the maturity of a selected domain based on a comprehensive set of criteria [2]. These models have progressive maturity levels, allowing the organization to plan how to reach higher maturity levels and to evaluate their outcomes on achieving that.

A maturity model is a framework that describes, for a specific area of interest, a set of levels of sophistication at which activities in this area can be carried out [1]. Essentially, maturity models can be used: to evaluate and compare organizations' current situation, identifying opportunities for optimization; to establish goals and recommend actions for increasing the capability of a specific area within an organization; and as an instrument for controlling and measuring the success of an action [3].

Product lifecycle mainly comprises several phases, e.g. research, development, production and operation/product support [4]. The development phase comprises the sub phases shown in Fig. 1: feasibility, concept, definition, development and series, which involve improvement and modifications. Product collaborative design encompasses all the processes before the production phase, and the information management strategy of products achieve internal information sharing and collaborative design by integrating data and knowledge throughout the whole product lifecycle and managing the completeness of the information in each stage of product design.

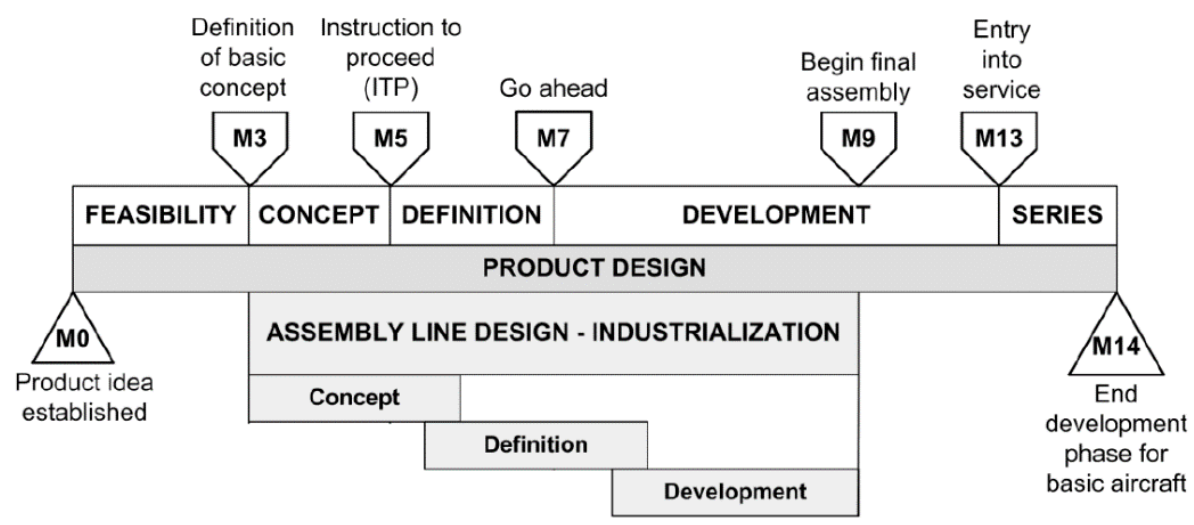

Fig. 1. Airbus product lifecycle and milestones development.

Researches on the product maturity are mainly about project management maturity which are used to evaluate and improve the project management capabilities of enterprises. A smaller part of the researches have discussed the concept of product maturity, and the number of works devoted to studying maturity of related processes and 
resources is insignificant. Wang et al. [5] proposed the concept of space product maturity and established a management model of product maturity, but it lacks the research about product maturity promoting the product development process. Tao and Fan [6] discussed the concept of maturity and management control method in the process of integration, but the division of the maturity level is not intuitive, and discussed little about application of product maturity in collaborative R\&D platform. Chen and Liu [7] provided the application of a strategy of product maturity for collaborative design on the collaborative development platform Teamcenter to verify the effectiveness and the controllability of the strategy. Wuest et al. [8] adapted the state gate model, a well-established methodology for product and software development, to production domain and indicated that it may provide valuable support for product and process quality improvement although the success is strongly dependent of the right adaptation.

The main objective of this paper is the design of a maturity management model for controlling the functional and industrial design phase of an aeronautical assembly line in the Airbus company (Fig. 1), and explores the development of this model in 3DExperience, a collaborative software platform by Dassault Systémes [9].

\section{Antecedents and iDMU concept}

The industrial Digital Mock-Up (iDMU) is the Airbus proposal to perform the design process with a unique team with a unique deliverable. The iDMU is defined by Airbus to facilitate the integration of the processes of the aircraft development on a common platform throughout all their service life. It is a way to help in making the functional and the industrial designs evolving jointly and collaboratively. An iDMU gathers all the product, processes and resources information to model and validate a virtual assembly line, and finally to generate the shopfloor documentation needed to execute the manufacturing processes [10-11].

Airbus promoted the Collaborative Engineering in the research project "Advanced Aeronautical Solutions Using PLM Processes and Tools" (CALIPSOneo) by implementing the iDMU concept [12-14]. The iDMU implementation was made for the industrialization of the A320neo Fan Cowl, a mid-size aerostructure. It was built by customizing CATIA/DELMIA V5 [9] by means of the PPR model concept. The PPR model of this commercial software provided a generic data structure that had to be adapted for the products, processes and resources of each particular implementation. In this case, a specific data structure was defined to support the Airbus products, the industrial design process, the process structure nodes, the resources structure nodes and their associated technological information, 3D geometry and metadata.

The process followed by Airbus to execute a pilot implementation of the iDMU is briefly described as follows. The previously existing Product structure was used and an ad-hoc application was developed that periodically updated all the modifications released by functional design. The Process and Resources structures were populated directly in the PPR context. The Process structure comprised four levels represented by four concepts: assembly line, station, assembly operation and task. Each concept 
has its corresponding constraints (precedence, hierarchy), its attributes and its allocation of products to be assembled and resources to be used. Once the PPR structures were defined, the system calculated the product digital mock-up and the resources digital mock-up that relate to each process node. As a result, the designer created simulations in the 3D graphical environment to analyse and validate the defined manufacturing solution. This validation of the process, product and resource design, by means of Virtual Manufacturing utilities in a common context, is a key feature in the Collaborative Engineering deployment.

The iDMU supports the collaborative approach through 3 main elements. First, it allows sharing different design perspectives, to reveal solutions that while valid for a perspective (e.g. resources design) cause problems in other perspectives (e.g. industrialization design), and to solve such issues. Second, it enables checking and validation of a high number of alternatives, allowing improving the harmonization and optimization of the design as a whole. And third, it is possible to reuse information contained in the iDMU by other software systems used in later stages of the lifecycle, facilitating the integration and avoiding problems with translation of models into intermediate formats, and making easier the use of new technologies such as augmented reality.

The CALIPSOneo project [12-14], with a scope limited to the A320neo fan cowl, allowed confirming that the iDMU provides a suitable platform to develop the sociotechnical process needed by the collaborative engineering. However, the project also revealed that the general functionalities provided by the adopted PLM commercial solution required an important research and development work to implement the data structures and functions needed to support the iDMU.

An important factor in the implementation of an iDMU is the need for a PLM tool capable of coordinating the workflow of all participants by means the definition and control of the lifecycle of allocated elements of the PPR structure, i.e. to manage its maturity states. At present, this issue is being addressed in the research project "Value Chain: from iDMU to Lean documentation for assembly" (ARIADNE).

\section{Methodology}

As said before, one of the studies carried out within the scope of the ARIADNE project was the analysis of capabilities that a PLM tool requires to manage the maturity states of the iDMU. Such a PLM tool aims the following objectives:

- To define independent and different maturity states sets for Product, Process and Resource revisions.

- To define precedence constraints between the maturity states of a Process revision, and the maturity states of its related Products and Resources.

- To define, for each Process revision maturity state, other conditions (e.g. attribute values) that are to be met prior to evolving a Process revision to the maturity state.

- To define, for each Process revision maturity state, that some process data or relations are not modifiable from this maturity state onwards.

- To display online, in the process revision iDMU, the Products and Resources evolved through maturities from the last time it was vaulted. 
- To display online, in the process revision iDMU, the impact of the evolved Products and Resources and how easy these issues can be fixed.

In order to prove the capabilities of a new PLM tool to meet these objectives, a simple lifecycle model is proposed. The model has only three possible maturity states for every element of the PPR structure: In Work, Frozen and Released. However, the importance of the proposed model lies in a set of constraints that prevent the promotion between maturity states, as described below. This simple model aims to be a preliminary test to evaluate a new PLM tool, so that it can be improved and extended with new states, relationships, constraints, rules, etc.

The In Work state is used for a new version of a product, process or resource element in the PPR structure. In Work data are fully modifiable and can be switched to Frozen by the owner, or to Released by the project leader. Frozen is an intermediate state between In Work and Released. It can be used, for example, for data waiting for approval. Frozen data are partially modifiable (for minor version changes) and can be switched back and forth between In Work and Frozen by the owner, or to Released by the project leader. Released is the final state of a PPR element, e.g. when a product is ready for production, a process is accepted for industrialization, or a resource is fully configured for its use. Released data cannot be deleted and cannot switched back to previous states.

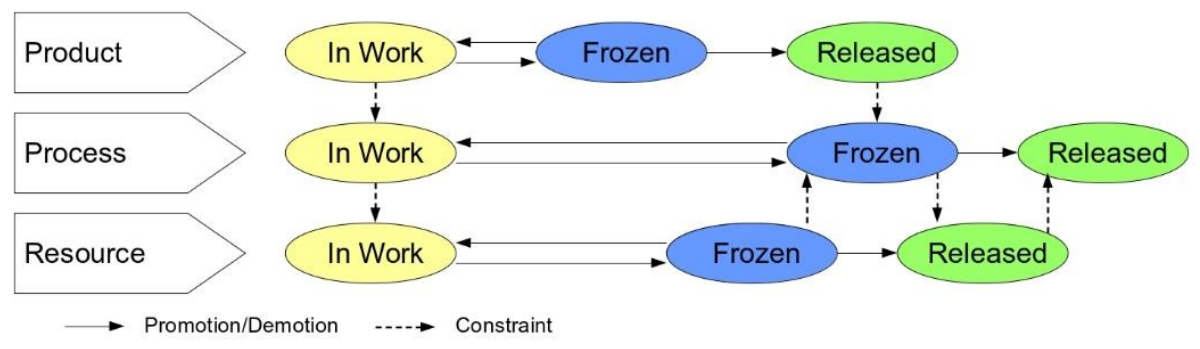

Fig. 2. Proposed simple model for the lifecycle of the PPR structure.

Fig. 2 shows a schema of the proposed model. At the beginning of the lifecycle, since Design Engineering starts the product design, usually Manufacturing Engineering can begin to plan the process, set up the layout and define the necessary resources. In this situation, all product, process and resource elements in the PPR structure are In Work. The collaborative environment must allow the visualization and query of information under development to the different actors of the system, based on roles and permissions, so that it helps to detect design errors and make right decisions.

The new PLM tool must provide a set of rules or constraints that allow to control and alert the designer about non-coherent situations. Fig. 2 schematically presents some constraints to promote a PPR element. For instance, it is not possible to assign to a process node a maturity state of Frozen until the related product node has a maturity state of Released and the allocated resource has a maturity state of Frozen. In a similar way, to promote a process to Released, the allocated resource must be in $R e$ - 
leased. On the other hand, the resource element can only reach the maturity state of Released when the process element has been Frozen previously.

In addition to define constraints between elements of different types (product, process and resource), it is necessary to establish rules between elements of the same type to control the change of maturity states of their interconnected elements. For instance, the following constraint inside the Product structure could be established: the designer of a product consisting of several parts can change the state of the product element to Frozen/Released when all its parts already have that same state, so that a part still unfinished (In Work) alerts him that the product cannot be promoted yet.

\section{$4 \quad$ Practical application}

The proposed model for managing the maturity states of an iDMU was implemented and tested in a PLM commercial software, within the frame of the ARIADNE project. The implementation was carried out with the 3DExperience software solution by Dassault Systémes.

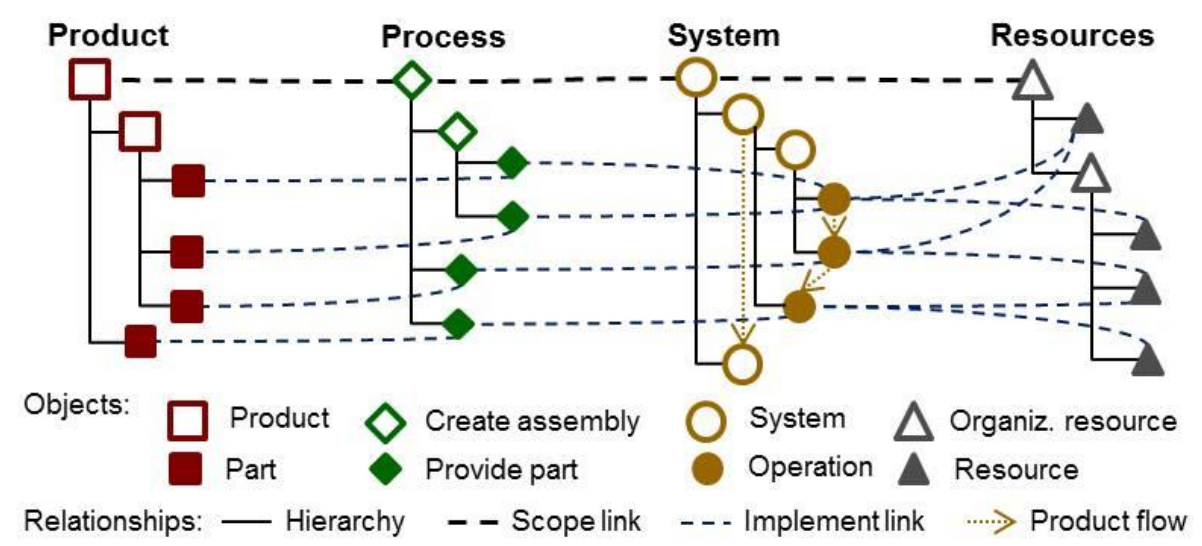

Fig. 3. Schema of implementation of Airbus iDMU concept in 3DExperience.

The PPR structure in 3DExperience differs slightly from CATIA/DELMIA V5 so that the process of building the iDMU is different from those developed in previous projects. A significant difference is that the previous 3-elements PPR structure is replaced by a 4-elements structure, as represented schematically in Fig. 3:

- Product: it presents the functional zone breakdown in an engineering oriented organization. It is modelled by Design Engineering to define the functional view for structure and system installation.

- Process: it is focused to model the process plan from a functional point of view. It is indeed a product structure composed of a cascade of components identified by part numbers that presents how the product is built and assembled. Thus, both product and process elements of the PPR structure are directly correlated. 
- System: it defines the work flow operation. It contains a set of system/operations that corresponds to the steps necessary to correlate with the Process structure. It contains the information necessary to perform operations such as balancing the assembly lines.

- Resource: it represents the layout design for a manufacturing plant. Resources can be classified as working (e.g. robot, worker, conveyor), non-working (e.g. tool device) or organizational (e.g. station, line). The required resources are attached to operations in the System structure, as shown in Fig. 3.

The adopted PLM software integrates a default lifecycle model to any created object that controls the various transitions in the life of the object. This model includes elements such as user roles, permissions, states and available state changes. To facilitate the collaborative work, 3DExperience also provides a lifecycle model to manage Engineering Changes, which has links to PPR objects, and a transfer ownership functionality that can be used to pass an object along to another authorized user to promote it. Both PPR and Engineering Changes lifecycle models can be customized. These characteristics made 3DExperience an adequate collaborative platform for the purpose of this work.

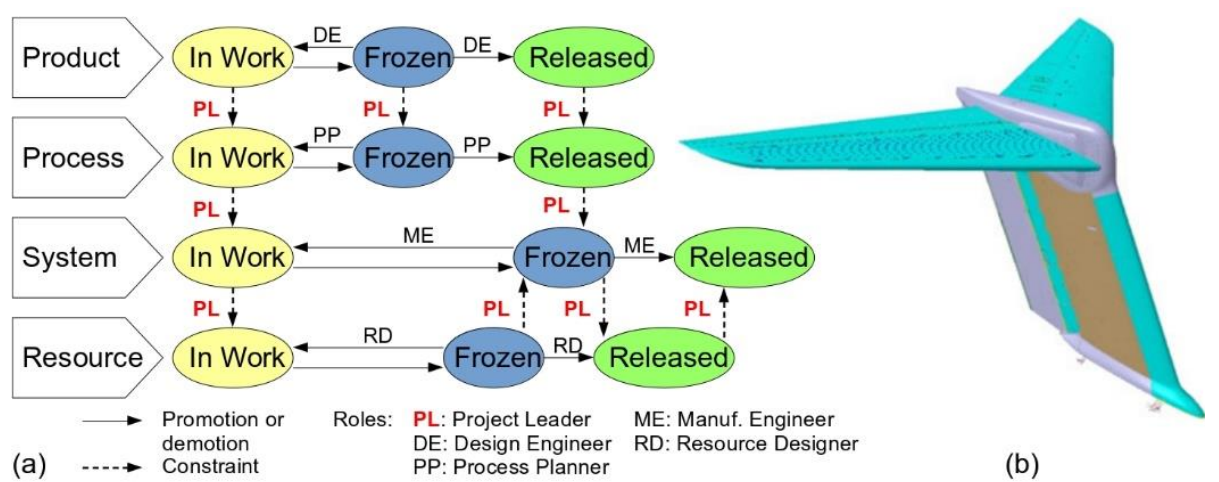

Fig. 4. (a) Extension of proposed model and (b) Airbus A400M empennage.

The objectives that a PLM tool must satisfy for managing the maturity states, described in the previous section, were analysed to fit the 4-element PPR structure of the 3DExperience software. Accordingly, the proposed model was redefined as shown in Fig. 4(a). As can be seen, the set of constraints for the System lifecycle is equivalent to the previous set of constraints for the Process lifecycle, whereas that Process elements are the bridge between Products and Systems.

A series of roles has been defined (see Fig. 4(a)) to implement the proposed model of maturity states in 3DExperience, such as the Project Leader (PL) and a different type of user to design each of the PPR structures: a Designer Engineer (DE), a Process Planner (PP), a Manufacturing Engineer (ME) and a Resources Designer (RD). Each system user is responsible for designing and promoting/demoting each node of its structure to the three possible states, as shown in Fig. 4(a). The PL coordinates all 
maturity state changes: he checks that there are no inconsistencies and gives the other users permission to make the changes.

Designers have several possibilities for building the iDMU using the 3DExperience graphical interface. Briefly, the maturity state is stored as an attribute of each PPR element, so it can be accessible from the query tool "Properties". The software also provides the "Team Maturity" utility to display information in the graphical environment about the maturity states. This utility displays a coloured flag in each element of the model tree that indicates its maturity state; however, it applies just for Product and Resource elements, i.e. elements that have associated geometry. Another utility allows displaying graphical information about the related elements of an allocated iDMU element. Both graphical utilities, for maturity states and related elements, were used to search and filter information before changing an object state. To promote or demote the maturity state of an iDMU element, the "Change Maturity" utility presents different fields with the available changing states and related information according to the lifecycle model, roles and permissions.

The Airbus A400M empennage (about 34000 parts, see Fig. 4(b)) and its assembly processes were selected to develop the iDMU in 3DExperience. The empennage model developed in CATIA V5 was used as the Product structure. Process, System and Resource structures were modelled from scratch. Different use cases were evaluated by choosing small and more manageable parts of the iDMU to change their maturity states in the collaborative platform. The following is a summary of the implementation process carried out. An example is shown in Fig. 5.

\begin{tabular}{|c|c|c|c|c|c|c|}
\hline \multirow{3}{*}{ Product } & & & & & & \\
\hline & & In Work & Frozen & In Work & Frozen & Released \\
\hline & & & $d$ & & 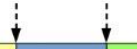 & \\
\hline \multirow[t]{2}{*}{ Process } & & In Work & Frozen & In Work & Frozen & Released \\
\hline & & & & & & $h$ \\
\hline \multirow[t]{2}{*}{ System } & & & In Work & & & Frozen Released \\
\hline & & & $e$ & $f$ & & $g$ \\
\hline Resource & & In Work & & Frozen In Work & Frozen & Released \\
\hline
\end{tabular}

Fig. 5. An example of implementation of the proposed simple model.

At the beginning of the lifecycle, the PL authorized all other system actors to work together in the iDMU at the same time in the collaborative platform (label $a$ in Fig. 5). The main PPR structures were created and scope links were established between them. In this situation, all PPR nodes were In Work while the iDMU was designed in a collaborative and coordinated way.

One of the first state changes in the iDMU is made by the DE when it promotes a component or subproduct to Frozen $(b)$. In this situation, only minor design changes could be made to the frozen component, which will have no impact on the rest of the iDMU (including other components of the product). Demoting the component to an In 
Work state $(c)$ would indicate that major changes are required as a result of the current design state in other areas of the iDMU. In general, the promotion to Released of every PPR structure will be carried out in an advanced state of the whole iDMU. This means that their design has been considered as stable and that no significant changes will occur that affect other parts of the iDMU.

Maturity state changes in the Process structure are conditioned by the state of related components in the Product structure. Thus, before promoting a Process element $(d)$, the PP must check the status of related components with the aforementioned 3DExperience utilities to search and analyse the related elements and their states of maturity. If the related product is Frozen/Released, the PP can request authorization to the PL to promote the Process element.

Another of the first state changes of maturity that occurs in the iDMU is that of resources. Thus, the RD promotes a resource to Frozen (e) or demotes it to In Work ( $f$ ) following the same guidelines as the DE with the products. Instead, the promotion of a resource to Released $(g)$ can only be authorized by the PL when the related assembly system is Frozen, indicating that the assembly line has been designed except for possible minor changes that would not affect the definition of the resources.

The ME is the last actor to promote the state of his work in the iDMU: the design of the assembly system/line. In order to freeze his work $(h)$, the ME needs to know in advance the final design of the product assembly process and also the definition of the necessary resources. Any changes in product or process structures, even if they are minor, could have a relevant impact on the definition of the assembly line. Therefore, the ME must previously verify that related assembly processes are Released and required resources are Frozen. Since resource nodes are linked to the System structure through operation nodes, the ME extensively uses the 3DExperience utilities to trace all affected nodes and check their maturity states. As discussed above, the promotion to Released of all PPR structures occurs in an advanced development of the iDMU, being the last two steps those relating to Resource and System structures.

\section{Conclusions}

This paper presents the methodology and preliminary results for the management in a collaborative environment of the maturity states of PPR elements with all product, process and resource information associated with the assembly of an aeronautical component. The methodology aims to evaluate the suitability of PLM tools to implement the Airbus methodology in the creation of a control mechanism that allows a collaborative work.

The proposed model shows in a simple way the importance of the flow of information among the different participants of a unique team to build an iDMU as the unique deliverable in a collaborative platform. An outstanding feature of the lifecycle model is its ability to authorize or restrict the promotion of a product, process or resource element depending on the states of the related elements. Different use cases with coherent and non-coherent situations have been successfully analysed using 3DExperience to implement an iDMU for the Airbus A400M empennage. 
In this work, the change management of maturity states has been coordinated by a Project Leader. The next step will be to customize 3DExperience to automate the maturity state changes, so that the system is responsible for evaluating the information of related elements, allowing or preventing the designer from promoting an iDMU element.

\section{Acknowledgements}

The authors wish to thank the Andalusian Regional Government and the Spanish Government for its financial support through the research project "Value Chain: from iDMU to Lean documentation for assembly" (ARIADNE). The work of master thesis students, Gonzalo Monguió and Andrés Soto, is also greatly acknowledged.

\section{$7 \quad$ References}

1. Alonso J, Martinez de Soria I, Orue-Echevarria L, Vergara M (2010). Enterprise Collaboration Maturity Model (ECMM): Preliminary Definition and Future Challenges. Enterprise Interoperability IV. Springer, London, pp 429-438.

2. De Bruin T, Freeze R, Kaulkarni U, Rosemann M (2005). Understanding the main phases of developing a maturity assessment model. In: Australasian Conference on Information Systems (ACIS). Sydney, Australia, pp 1-11.

3. Hain S (2010). Developing a Situational Maturity Model for Collaboration (SiMMCo) Measuring Organizational Readiness. St. Gallen, Switzerland, pp 1-6.

4. Wellsandt S, Nabati E, Wuest T, Hribernik K, Thoben K-D (2016). A survey of product lifecycle models: Towards complex products and service offers. Int J PLM 9(4):353-390.

5. Wang W, Zhu X, Li Y (2007). Research on Space Product Maturity and Application. Aerospace Industry Management 7:26-31.

6. Tao J, Fan Y (2006). Application of Maturity in Development of Aircraft Integrated Process. J Beijing Univ Aeronautics and Astronautics 32:1117-1120.

7. Chen M, Liu J (2013). Maturity Management Strategy for Product Collaborative Design. Advanced Materials Research 712-715:2856-2860.

8. Wuest T, Liu A, Lu S C-Y, Thoben K-D (2014). Application of the stage gate model in production supporting quality management. Procedia CIRP 17:32-37

9. Dassault Systemés. https://www.3ds.com

10. Menéndez JL, Mas F, Serván J, Ríos J (2012). Virtual verification of the AIRBUS A400M final assembly line industrialization. AIP Conf Proc 1431(1):641-648.

11. Mas F, Menéndez JL, Oliva M, Ríos J (2013). Collaborative Engineering: An Airbus Case Study. Procedia Eng 63:336-345.

12. Mas F, Menéndez JL, Oliva M, Gómez A, Ríos J (2013). Collaborative Engineering Paradigm Applied to the Aerospace Industry. In: Bernard A, Rivest L, Dutta D, editors. Product Lifecycle Management for Society. Springer Berlin Heidelberg, pp 675-684.

13. Mas F, Menéndez JL, Oliva M, Ríos J, Gómez A, Olmos V (2014). iDMU as the Collaborative Engineering engine: Research experiences in Airbus. In: 2014 International Conference on Engineering, Technology and Innovation (ICE), pp 1-7.

14. Mas F, Oliva M, Ríos J, Gómez A, Olmos V, García JA (2015). PLM Based Approach to the Industrialization of Aeronautical Assemblies. Procedia Eng 132:1045-1052. 